\title{
COMPOSITION OF LINEAR FRACTIONAL TRANSFORMATIONS IN TERMS OF TAIL SEQUENCES
}

\author{
LISA JACOBSEN
}

ABSTRACT. We consider sequences $\left\{s_{n}\right\}$ of linear fractional transformations. Connected to such a sequence is another sequence $\left\{S_{n}\right\}$ of linear fractional transformations given by

$$
S_{n}=s_{1} \circ s_{2} \circ \cdots \circ s_{n}, \quad n=1,2,3, \ldots
$$

We introduce a new way of representing $s_{n}$ (in terms of so-called tail sequences). This representation is established to give nice expressions for $S_{n}$. It can be seen as a generalization of the canonical form for $s_{n}$, which gives nice expressions for

$$
T_{k}=\underbrace{s_{n} \circ s_{n} \circ \cdots \circ s_{n}}_{(k \text { terms })} .
$$

1. Introduction. A linear fractional transformation

$$
s(w)=\frac{a+c w}{b+d w}, \quad a, b, c, d \in \mathbf{C}, \Delta=a d-b c \neq 0,
$$

is a meromorphic function which maps the extended complex plane $\hat{\mathbf{C}}=\mathbf{C} \cup\{\infty\}$ bijectively onto itself. Hence, we adopt the convention that

$$
a+c w=a \quad \text { if } c=0 \text { and } w=\infty,
$$

and

$$
b+d w=b \quad \text { if } d=0 \text { and } w=\infty .
$$

(It should be noted that the notation (1.1) used in this paper differs from the standard notation $(a w+b) /(c w+d)$.) If $s(w) \not \equiv w$, then $s$ has two fixed points, $x$ and $y$, which coincide iff $s$ is parabolic. It is well known that then

$$
\frac{s(w)-x}{s(w)-y}=\frac{b+d y}{b+d x} \cdot \frac{w-x}{w-y} \quad \text { if } x \neq y,
$$

and

$$
\frac{1}{s(w)-y}=\frac{2 d}{b+c}+\frac{1}{w-y} \text { if } x=y \text {. }
$$

(We define

$$
w-y=0 \quad \text { if } w=y=\infty, \quad w-x=0 \quad \text { if } w=x=\infty .)
$$

Received by the editors July 31, 1984 and, in revised form, April 18, 1985.

1980 Mathematics Subject Classification. Primary 30D99; Secondary 30B70, 40A15. 
Clearly, $x$ and $y$ are both finite iff $d \neq 0$. Hence, if $d \neq 0$, then (1.4)-(1.5) is an alternative way to write (1.1). The following example illustrates one of the advantages obtained by this way of representing $s$.

EXAMPLE 1.1. Given the linear fractional transformation (1.1) with $d \neq 0$ and fixed points $x$ and $y(s(w) \not \equiv w$ since $d \neq 0)$, the composition of $n$ such transformations,

$$
S_{n}(w)=s \circ s \circ \cdots \circ s(w), \quad n \in \mathbf{N},
$$

is also a linear fractional transformation. If $s$ is nonparabolic, then $x \neq y$, and by (1.4)

$$
\begin{aligned}
\frac{S_{n}(w)-x}{S_{n}(w)-y} & =\frac{s\left(S_{n-1}(w)\right)-x}{s\left(S_{n-1}(w)\right)-y}=\frac{b+d y}{b+d x} \cdot \frac{S_{n-1}(w)-x}{S_{n-1}(w)-y} \\
& =\cdots=\left(\frac{b+d y}{b+d x}\right)^{n} \cdot \frac{w-x}{w-y}, \quad n \in \mathbf{N} .
\end{aligned}
$$

If $s$ is parabolic, then by (1.5)

$$
\begin{aligned}
\frac{1}{S_{n}(w)-y} & =\frac{1}{s\left(S_{n-1}(w)\right)-y}=\frac{2 d}{b+c}+\frac{1}{S_{n-1}(w)-y} \\
& =\cdots=n \cdot \frac{2 d}{b+c}+\frac{1}{w-y}, \quad n \in \mathbf{N} .
\end{aligned}
$$

From this we easily get $S_{n}(w)$ in closed form.

Let $\left\{s_{n}(w)\right\}_{n=1}^{\infty}$ be a sequence of linear fractional transformations and consider the composition

$$
S_{n}(w)=s_{1} \circ s_{2} \circ \cdots \circ s_{n}(w), \quad n \in \mathbf{N} .
$$

If we now try to use (1.4)-(1.5) as in Example 1.1, the expressions get complicated, even for quite small $n \in \mathbf{N}$, except in special cases such as (1.7).

In this paper we introduce a generalization of (1.4)-(1.5) which is adapted to this more general situation. It is based on a new concept, tail sequences, introduced in $\S 2$. These tail sequences generalize the fixed points in a certain sense. As an example we show in $\S 3$, that these formulas apply to continued fractions.

2. Tail sequences. Given the sequence

$$
s_{n}(w)=\frac{a_{n}+c_{n} w}{b_{n}+d_{n} w}, \quad \Delta_{n}=a_{n} d_{n}-c_{n} b_{n} \neq 0, \quad \text { for } n=1,2,3, \ldots
$$

Let us introduce the following concept:

DEFINITION 2.1. $\left\{u_{n}\right\}_{n=0}^{\infty}, u_{n} \in \hat{\mathbf{C}}$, is called a tail sequence for $\left\{s_{n}(w)\right\}_{n=1}^{\infty}$, if

$$
u_{n-1}=s_{n}\left(u_{n}\right) \text { for } n=1,2,3, \ldots \text {. }
$$

This concept has already been introduced for another purpose, for the special case where $\left\{s_{n}(w)\right\}$ is a continued fraction generating sequence, i.e., $c_{n}=0$ and $d_{n}=1$ for all $n[6]$. We refer to $\$ 3$ for information explaining the name "tail sequences". 
The following properties of tail sequences are proved by straightforward computation (we omit the proofs here):

Proposition 2.2. Let $\left\{u_{n}\right\}_{n=0}^{\infty}$ and $\left\{v_{n}\right\}_{n=0}^{\infty}$ be two tail sequences for the sequence $\left\{s_{n}(w)\right\}_{n=1}^{\infty}$ of linear fractional transformations. Then

A.

$$
u_{n} \neq v_{n} \text { for an } n \in \mathbf{N} \cup\{0\} \Rightarrow u_{n} \neq v_{n} \text { for all } n \in \mathbf{N} \cup\{0\}
$$

B.

$$
\begin{aligned}
& \frac{1}{s_{n}(w)-u_{n-1}}=\frac{d_{n}\left(b_{n}+d_{n} u_{n}\right)}{-\Delta_{n}}+\frac{\left(b_{n}+d_{n} u_{n}\right)^{2}}{-\Delta_{n}} \cdot \frac{1}{w-u_{n}}, \quad w \in \hat{\mathbf{C}}, \\
& \text { if } u_{n-1} \neq \infty \text { and } u_{n} \neq \infty
\end{aligned}
$$

C.

$$
\begin{aligned}
\frac{s_{n}(w)-u_{n-1}}{s_{n}(w)-v_{n-1}}=\frac{b_{n}+d_{n} v_{n}}{b_{n}+d_{n} u_{n}} & \frac{w-u_{n}}{w-v_{n}}, \quad w \in \hat{\mathbf{C}}, \\
& \text { if } u_{n}, u_{n-1}, v_{n}, v_{n-1} \neq \infty \text { and } u_{0} \neq v_{0} .
\end{aligned}
$$

By repeated use of parts B and C we get the following representation of $S_{n}\left(S_{n}\right.$ defined by (1.10)).

THEOREM 2.3. Let $\left\{u_{n}\right\}$ and $\left\{v_{n}\right\}$ be two distinct tail sequences for the sequence $\left\{s_{n}(w)\right\}_{n=1}^{\infty}$ of linear fractional transformations. Further, let

$$
S_{n}(w)=s_{1} \circ s_{2} \circ \cdots \circ s_{n}(w) \text { for all } n \in \mathbf{N} \text {. }
$$

If $u_{n} \neq \infty$ for $n=0,1, \ldots, N$, then

$$
\begin{aligned}
\frac{1}{S_{N}(w)-u_{0}}= & \sum_{j=1}^{N} d_{j} \frac{b_{j}+d_{j} u_{j}}{-\Delta_{j}} \prod_{k=1}^{j-1} \frac{\left(b_{k}+d_{k} u_{k}\right)^{2}}{-\Delta_{k}} \\
& +\frac{1}{w-u_{N}} \prod_{k=1}^{N} \frac{\left(b_{k}+d_{k} u_{k}\right)^{2}}{-\Delta_{k}}
\end{aligned}
$$

for all $w \in \hat{\mathbf{C}}$. If, in addition, $v_{n} \neq \infty$ for $n=0,1, \ldots, N$, then

$$
\frac{S_{N}(w)-u_{0}}{S_{N}(w)-v_{0}}=\frac{w-u_{N}}{w-v_{N}} \prod_{j=1}^{N} \frac{b_{j}+d_{j} v_{j}}{b_{j}+d_{j} u_{j}} \quad \text { for all } w \in \hat{\mathbf{C}} \text {. }
$$

Again the proofs are straightforward and omitted here.

From (2.3) or (2.4) we can find a closed expression for $S_{n}(w)$. On the other hand, to obtain some advantage of these formulas, we need to know at least one tail sequence, $\left\{u_{n}\right\}, u_{n} \neq \infty$ for $\left\{s_{n}(w)\right\}$. We shall see some examples.

EXAMPLE 2.4. Let $s_{n}=s$ for all $n \in \mathbf{N}$, where $s$ is given by (1.1) with $d \neq 0$. If $x$ is a fixed point of $s(w)$, then $\left\{u_{n}\right\}$ with $u_{n}=x$ for all $n$ is a tail sequence for $\left\{s_{n}(w)\right\}$. (All tail sequences $\left\{v_{n}\right\}$ for $\left\{s_{n}(w)\right\}$, where $v_{0}$ is not a fixed point of $s$, are nonconstant.) If $s$ has two distinct fixed points, $x$ and $y$ (finite since $d \neq 0$ ), then the choice $u_{n}=x$ and $v_{n}=y$ in (2.4) gives (1.8). If $s$ has only one fixed point $y$ ( $s$ 
is parabolic), then the choice $u_{n}=y$ in (2.3) reduces to (1.9), since then

$$
(b-c)^{2}+4 a d=0 \text { and } y=(c-b) / 2 d .
$$

This explains why Proposition 2.2C and B represent a generalization of (1.4)-(1.5).

EXAMPLE 2.5. Let $s_{2 n-1}=s_{1}$ and $s_{2 n}=s_{2}$ for all $n \in \mathbf{N}$. Further, let $x_{2 n}=x_{0}$ and $y_{2 n}=y_{0}$ be two fixed points of $S_{2}=s_{1} \circ s_{2}$, where $x_{0} \neq y_{0}$ if $S_{2}$ is nonparabolic. Then $x_{2 n+1}=x_{1}=s_{2}\left(x_{0}\right)$ and $y_{2 n+1}=y_{1}=s_{2}\left(y_{0}\right)$ are fixed points of the linear fractional transformation $\left(s_{2} \circ s_{1}\right)$, and $\left\{x_{n}\right\}$ and $\left\{y_{n}\right\}$ are tail sequences for $\left\{s_{n}(w)\right\}$.

This procedure of finding periodic tail sequences can easily be extended to the case where $\left\{s_{n}(w)\right\}$ is periodic with period $k \in \mathbf{N}$. On the other hand we do not gain much compared to (1.8) or (1.9), since we then have

$$
S_{k n+p}(w)=S_{k} \circ S_{k} \circ \cdots \circ S_{k} \circ S_{p}(w) \text { for } n \in \mathbf{N} \text { and } 0 \leqslant p \leqslant k .
$$

EXAMPLE 2.6. Let $\left\{u_{n}\right\}_{n=0}^{\infty}, u_{n} \in \mathbf{C}$, be given. Then there exist infinitely many sequences $\left\{s_{n}(w)\right\}_{n+1}^{\infty}$ of linear fractional transformations such that $\left\{u_{n}\right\}$ is a tail sequence for $\left\{s_{n}(w)\right\}$. If, for instance, $u_{n} \neq 0$ for all $n$, then $\left\{u_{n}\right\}$ is a tail sequence for $\left\{2 u_{n} u_{n-1} /\left(u_{n}+w\right)\right\}$ and for $\left\{u_{n-1}\left(1+u_{n}\right) /(1+w)\right\}$ etc.

In some situations it suffices to find the approximate location of $S_{n}(D)$ for some set $D \subseteq \mathbf{C}$. This can for instance be the case if we want to prove convergence results for $\left\{S_{n}(w)\right\}_{n=1}^{\infty}$ and estimate the truncation error for a given $n$.

EXAMPLE 2.7. Let

$$
s_{n}(w)=\frac{a_{n}}{1+w}=\frac{a+\varepsilon_{n}}{1+w} \quad \text { for all } n \in \mathbf{N},
$$

where $a, \varepsilon_{n} \in \mathbf{C}$ are chosen such that $\Delta_{n}=a_{n} \neq 0$ and $s(w)=(a+c w) /(b+d w)$ $=a /(1+w)$ is a hyperbolic or loxodromic linear fractional transformation (i.e., $a \neq 0, a \notin(-\infty,-1 / 4])$. Let $x$ be the attractive fixed point of $s$, and $y$ the repulsive one. Then $x, y \neq \infty$ and by (1.4) $|b+d x|>|b+d y|=|c-d x|$, i.e., here $|1+x|>$ $|x|$. Let $D=|b+d x|-|c-d x|$, i.e., here $D=|1+x|-|x|$, and assume that

$$
\left|\varepsilon_{n}\right| \leqslant\left(D^{2}-\mu^{2}\right) / 4 \text { for all } n, 0<\mu \leqslant D .
$$

Then it is possible to prove by straightforward computation that

$$
s_{n}(V) \subseteq V \text { and } S_{n}(V) \rightarrow\{u\} \subset V \text { for } V=\left\{z \in \mathbf{C} ;|z-x| \leqslant \frac{D-\mu}{2}\right\},
$$

for a $u \in V$, and

$$
s_{n}^{-1}(W) \subseteq W \text { for } W=\left\{z \in \mathbf{C} ;|z-y| \leqslant \frac{D-\mu}{2}\right\} .
$$

(See [1].) Let $\left\{u_{n}\right\}$ be the tail sequence for $\left\{s_{n}(w)\right\}$ with $u_{0}=u$. Then $u_{n} \in V$ for all $n$, because the point

$$
\left\{u_{n}\right\}=\lim _{m \rightarrow \infty} s_{n+1} s_{n+2} \cdots s_{n+m}(V) \subseteq V \text { for all } n .
$$


Moreover, let $\left\{v_{n}\right\}$ be a tail sequence for $\left\{s_{n}(w)\right\}$ with $v_{0} \in W$. Then $v_{n} \in W$ for all $n$, since $v_{n}=s_{n}^{-1}\left(v_{n-1}\right)$. From (2.4) we then get

$$
\frac{S_{n}(w)-u_{0}}{S_{n}(w)-v_{0}}=K_{n} \frac{w-u_{n}}{w-v_{n}} \quad \text { for all } w \in \hat{\mathbf{C}}
$$

where

$$
\begin{aligned}
\left|K_{n}\right| & =\prod_{j=1}^{n}\left|\frac{1+v_{j}}{1+u_{j}}\right| \leqslant \prod_{j=1}^{n} \frac{|1+y|+(D-\mu) / 2}{|1+x|-(D-\mu) / 2} \\
& =\left(\frac{|1+x|+|x|-\mu}{|1+x|+|x|+\mu}\right)^{n} .
\end{aligned}
$$

Since $u_{n}, v_{n}, u_{0}, v_{0} \neq \infty$ and $K_{n} \rightarrow 0$, we can also solve (2.9) to get

$$
S_{n}(w)=\frac{w\left(u_{0}-K_{n} v_{0}\right)-\left(u_{0} v_{n}-K_{n} v_{0} u_{n}\right)}{w\left(1-K_{n}\right)-\left(v_{n}-K_{n} u_{n}\right)} \sim \frac{u_{0}\left(w-v_{n}\right)}{w-v_{n}} .
$$

This means in particular that $S_{n}(w) \rightarrow u_{0}$ for all $w \in \mathbf{C} \backslash W$ (which is not a new result; see the next section).

By the same method as introduced in Example 2.7, we also get estimates for $K_{n}$ in (2.9) in more general cases. For instance, if

$$
s_{n}(w)=\frac{a+\varepsilon_{n}+\left(c+\lambda_{n}\right) w}{b+d w}, \quad d>0, \Delta_{n} \neq 0
$$

where $s$ is a hyperbolic or loxodromic linear fractional transformation, then (2.7) and (2.8) are valid for

$$
V=\left\{z \in \mathbf{C} ;|z-x| \leqslant \frac{D-\mu}{2 d}\right\}, \quad W=\left\{z \in \mathbf{C} ;|z-y| \leqslant \frac{D-\mu}{4 d}\right\},
$$

if

$$
\left|\lambda_{n}\right|+2 d \frac{\left|\varepsilon_{n}+x \lambda_{n}\right|}{D-\mu} \leqslant \frac{D-\mu}{2}, \quad\left|\lambda_{n}\right| \leqslant \frac{D-\mu}{4},
$$

and

$$
\left|\varepsilon_{n}+y \lambda_{n}\right| \leqslant \frac{D^{2}-\mu^{2}}{8 d} \text { for all } n
$$

This gives

$$
\begin{aligned}
\left|K_{n}\right| & =\prod_{j=1}^{n}\left|\frac{b+d v_{j}}{b+d u_{j}}\right| \leqslant\left(\frac{|b+d y|+(D-\mu) / 4}{|b+d x|-(D-\mu) / 2}\right)^{n} \\
& \leqslant\left(\frac{|b+d x|+|c-d x|-\mu}{|b+d x|+|c-d x|+\mu}\right)^{n} \text { for all } n .
\end{aligned}
$$

For $s_{n}(w)=\left(a+\varepsilon_{n}\right) /\left(b+\delta_{n}+w\right)$, see [2]. For even more general examples where $V$ and $W$ vary with $n$, see [ 2 and 3 ]. 
3. Application to continued fractions. A continued fraction

$$
K \frac{a_{n}}{b_{n}}=\frac{a_{1}}{b_{1}}+\frac{a_{2}}{b_{2}}+\cdots=\frac{a_{1}}{b_{1}+\frac{a_{2}}{b_{2}+\frac{a_{3}}{b_{3}}+}}, \quad a_{n} \neq 0
$$

$a_{n}, b_{n} \in \mathbf{C}$, is an infinite process, where a sequence of approximants,

$$
f_{n}=\frac{a_{1}}{b_{1}}+\frac{a_{2}}{b_{2}}+\cdots+\frac{a_{n}}{b_{n}} \text { for } n=1,2,3, \ldots
$$

is generated from the ordered pair $\left(\left\{a_{n}\right\}_{n=1}^{\infty},\left\{b_{n}\right\}_{n=1}^{\infty}\right)$. We say that $K\left(a_{n} / b_{n}\right)$ converges and has the value $f$ if $\lim f_{n}=f$ exists in $\hat{\mathbf{C}}$. Defining the linear fractional transformations

$$
s_{n}(w)=\frac{a_{n}}{b_{n}+w}, \quad\left(\Delta_{n}=a_{n} \neq 0\right) \quad \text { for } n=1,2,3, \ldots,
$$

we see that $f_{n}=S_{n}(0)$, where $S_{n}$ is given by (1.10).

If $K\left(a_{n} / b_{n}\right)$ converges, then so do also all its tails

$$
\stackrel{n}{K=1}_{n=1}^{\infty} \frac{a_{m+n}}{b_{m+n}}=\frac{a_{m+1}}{b_{m+1}}+\frac{a_{m+2}}{b_{m+2}}+\cdots \quad \text { for } m=0,1,2, \ldots
$$

Let $f^{(m)}$ denote the value of the $m$ th tail (3.4). Then, clearly, $f^{(n-1)}=a_{n} /\left(b_{n}+f^{(n)}\right)$, that is, $\left\{f^{(n)}\right\}$ is a tail sequence for $\left\{s_{n}(w)\right\}$ or for $K\left(a_{n} / b_{n}\right)$. This is what inspired Waadeland when choosing a name for sequences connected with $K\left(a_{n} / b_{n}\right)$ satisfying (2.2) [6].

It is easy to prove that when $s_{n}$ has the form (3.3), then $S_{n}$ has the form

$$
S_{n}(w)=\frac{A_{n}+A_{n-1} w}{B_{n}+B_{n-1} w} \quad \text { for } n=1,2,3, \ldots,
$$

where $\left\{A_{n}\right\}$ and $\left\{B_{n}\right\}$ satisfy the recursion

$$
Y_{n}=b_{n} Y_{n-1}+a_{n} Y_{n-2} \text { for } n=1,2,3, \ldots,
$$

with initial values $A_{-1}=B_{0}=1$ and $A_{0}=B_{-1}=0$. This means that the tail sequence $\left\{-h_{n}\right\}$ of $K\left(a_{n} / b_{n}\right)$ with $-h_{0}=\infty$, can be written

$$
-h_{n}=S_{n}^{-1}(\infty)=-\frac{B_{n}}{B_{n-1}}=-b_{n}-\frac{a_{n}}{b_{n-1}}+\frac{a_{n-1}}{b_{n-2}}+\cdots+\frac{a_{2}}{b_{1}}, \quad n \in \mathbf{N} .
$$

This tail sequence is of particular importance in generating convergence results for continued fractions. From Theorem 2.3 we get the following corollary:

COROllary 3.1. Let $\left\{u_{n}\right\}$ and $\left\{v_{n}\right\}$ be two distinct tail sequences of the continued fraction $K\left(a_{n} / b_{n}\right)$. If $u_{n}, v_{n} \neq \infty$ for $n=0,1, \ldots, N$, then

$$
\begin{aligned}
S_{N}(0) & =u_{0} \frac{1-\prod_{j=1}^{N} u_{j} / v_{j}}{1-\prod_{j=0}^{N} u_{j} / v_{j}}=u_{0} v_{0} \frac{\prod_{j=1}^{N} v_{j}-\prod_{j=1}^{N} u_{j}}{\prod_{j=0}^{N} v_{j}-\prod_{j=0}^{N} u_{j}} \\
& =u_{0}-u_{0}\left(\sum_{m=1}^{N+1} \prod_{j=1}^{m-1}\left(-\frac{b_{j}+u_{j}}{u_{j}}\right)\right)^{-1}
\end{aligned}
$$


and

$$
\begin{aligned}
h_{N} & =-v_{N} \frac{1-\prod_{j=0}^{N} u_{j} / v_{j}}{1-\prod_{j=0}^{N-1} u_{j} / v_{j}}=-\frac{\prod_{j=0}^{N} v_{j}-\prod_{j=0}^{N} u_{j}}{\prod_{j=0}^{N-1} v_{j}-\prod_{j=0}^{N-1} u_{j}} \\
& =-v_{N}-v_{N}\left(\sum_{m=1}^{N} \prod_{j=m}^{N}\left(-\frac{v_{j}}{b_{j}+v_{j}}\right)\right)^{-1} .
\end{aligned}
$$

Proof. $S_{N}(w)$ and $S_{N}^{-1}(w)$ are found by use of Theorem 2.3. We then use that $a_{n}=u_{n-1}\left(b_{n}+u_{n}\right)=v_{n-1}\left(b_{n}+v_{n}\right)$ for $n=1,2, \ldots, N$.

REMARK. If $u_{n} \neq \infty$, then $u_{n-1} \neq 0$ (if $n \geqslant 1$ ) and $u_{n+1} \neq-b_{n+1}$.

EXAMPLE 2.7 (CONTINUED). Let $s_{n}$ be defined as earlier. (That is, by (2.5) and such that the conditions mentioned hold.) Then $\left\{S_{n}(0)\right\}_{n=1}^{\infty}$ are the approximants of the continued fraction $K\left(a_{n} / 1\right)$. If (2.6) holds, then $\left\{u_{n}\right\}$ and $\left\{v_{n}\right\}$ as defined earlier, are two distinct tail sequences for $\left\{s_{n}(w)\right\}$, that is for $K\left(a_{n} / 1\right)$. Since $u_{n} \in V$ and $v_{n} \in W$ for all $n$, it follows that

$$
\left|\frac{u_{j}}{v_{j}}\right| \leqslant \frac{|x|+\frac{D-\mu}{2}}{|y|-\frac{D-\mu}{2}}=\frac{|1+x|+|x|-\mu}{|1+x|+|x|+\mu}=r<1 .
$$

This means that $\prod_{j=1}^{\infty}\left(u_{j} / v_{j}\right)$ diverges to 0 , and thereby that $\left\{S_{N}(0)\right\}$ converges to $u_{0}$ by Corollary 3.1. Hence we have proved a useful criterion for convergence of continued fractions. (This criterion is not a new result. It was proved in a different form and by a different method by Perron [4, Satz 2.40]. Later, Scott and Wall proved the parabola theorem [5] which extends this result considerably.)

This method can be used to develop new convergence criteria also. For instance, if

$$
s_{n}(w)=\frac{a_{n}+\varepsilon_{n}}{1+w} \text { for } n=1,2,3, \ldots
$$

where $\left\{a_{n}\right\}$ is periodic with period length $k \in \mathbf{N}$, and the linear fractional transformation

$$
S(w)=\frac{a_{1}}{1}+\frac{a_{2}}{1}+\cdots+\frac{a_{k}}{1+w}
$$

is hyperbolic or loxodromic, the argument can be copied to prove that if $\left|\varepsilon_{n}\right| \leqslant$ some upper bound, then $K\left(\left(a_{n}+\varepsilon_{n}\right) / 1\right)$ converges [1]. More general results are shown in [3].

Recently, modified approximants, $S_{n}\left(w_{n}\right)$, where the $n$th tail of $K\left(a_{n} / b_{n}\right)$ is replaced by a modifying factor $w_{n} \in \hat{\mathbf{C}}$, have partly replaced the ordinary approximants $f_{n}=S_{n}(0)$ in special cases. Furthermore, tail sequences $\left\{S_{n}^{-1}\left(u_{0}\right)\right\}$, where $u_{0}$ is not necessarily $\infty$ or the value of the continued fraction (if it converges), are found to be of interest. Clearly, Theorem 2.3 can be used for these approximants and tail sequences as well. 


\section{REFERENCES}

1. L. Jacobsen, Some periodic sequences of circular convergence regions, Lecture Notes in Math., vol. 932. Springer-Verlag, 1981, pp. 87-98.

2. Modified approximants for continued fractions. Construction and applications, Kgl. Norske Vid. Selsk. Skr. 3 (1983), 1-46.

3. __ Nearness of continued fractions, Math. Scand. (to appear).

4. O. Perron, Die Lehre von den Kettenbrüchen, Vol. II, Teubner, Stuttgart, 1957.

5. W. T. Scott and H. S. Wall, A convergence theorem for continued fractions, Trans. Amer. Math. Soc. 47 (1940), 155-172.

6. H. Waadeland, Tales about tails, Proc. Amer. Math. Soc. 90 (1984), 57-64.

Department of Mathematics and Statistics, University of Trondheim AVH, 7055 DragVoll, NORWAY 